\section{Pneumothorax in an early phase after allogeneic hematopoietic stem cell transplantation}

\author{
Yasuhiro Ebihara, ${ }^{1}$ Shohei Yamamoto, ${ }^{1}$ \\ Shinji Mochizuki, ${ }^{2}$ Masao Tsukada, ${ }^{3}$ \\ Yuki Taya, ${ }^{3}$ Toshiro Kawakita, ${ }^{3}$ \\ Seiko Kato, ${ }^{3}$ Jun Ooi, ${ }^{3}$ \\ Satoshi Takahashi, ${ }^{3}$ Arinobu Tojo, ${ }^{3}$ \\ Kohichiro Tsuji 1,2,4 \\ 1Department of Pediatric \\ Hematology/Oncology, Research \\ Hospital, The Institute of Medical \\ Science, The University of Tokyo; \\ ${ }^{2}$ Division of Stem Cell Processing, Center \\ for Stem Cell Biology and Regenerative \\ Medicine, The Institute of Medical \\ Science, The University of Tokyo; \\ ${ }^{3}$ Department of Hematology/Oncology, \\ Research Hospital, The Institute of \\ Medical Science, The University of Tokyo; \\ ${ }^{4}$ Department of Pediatrics, Shinshu Ueda \\ Medical Center, Japan
}

\section{Abstract}

Pneumothorax is very rare after early phase of hematopoietic stem cell transplantation (HSCT) and usually accompanied with pulmonary chronic graft-versus-host disease (GVHD), such as bronchiolitis obliterans and bronchiolitis obliterans organizing pneumonia. The present study describes the case of a seventeen-year-old male diagnosed with acute myeloid leukemia who underwent allogeneic bone marrow transplantation (BMT). Pneumothorax occurred at day 43 after BMT. Pneumothorax occurred in early phase of HSCT is extremely rare. The early onset of acute GVHD and the entity of cytomegalovirus might worsen the pulmonary tissue damages for the onset of pneumothorax, indicating that we should be aware of the possibility to occur pneumothorax even in the early period after allogeneic HSCT.

\section{Introduction}

Allogeneic hematopoietic stem cell transplantation (HSCT) is currently a curative treatment option for many hematologic diseases. These recipients are at a high risk for pulmonary complications, which occur in $40-60 \%$ of transplanted recipients, and account for approximately $10-40 \%$ of transplant-related death. ${ }^{1-3}$ In the pulmonary complications, pneumothorax is rare, and most cases of pneumothorax reported previously were accompanied with pulmonary chronic graft-versus-host disease (cGVHD) in a late phase after HSCT. ${ }^{4-10}$ Here, we described a case of pneumothorax which occurred in an early phase after allogeneic HSCT.

\section{Case Report}

A 17 year-old male was diagnosed as acute myeloid leukemia with T lymphoblastic lymphoma, which was transformed from 8p11 myeloproliferative syndrome with chimeric CEP110-FGFR1 fusion transcript. Because of the absence of HLA-matched related or unrelated donor, he underwent myeloablative allogeneic bone marrow transplantation (BMT) from HLA-DR 1 locus mismatched unrelated female donor. On day 16 after BMT, grade III acute GVHD (aGVHD: skin, stage 3; liver, stage 0 ; gut, stage 2) developed. Then he received prednisolone (PSL) in addition to tacrolimus for GVHD prophylaxis. Twelve days after using PSL and tacrolims, aGVHD was almost improved. The engraftment of neutrophils was observed on day 22. Hematological complete remission and complete chimera in BM were obtained on day 31 , but since chimeric CEP110-FGFR1 fusion transcript still remained in real-time quantitative polymerase chain reaction analysis, immunosuppressant were tapered. On day 33, cytomegalovirus (CMV) pp65 antigenemia assay showed positive in his blood. Then he received ganciclovir (GCV), but the CMV positive cells still existed in his blood on day 40 even though he did not manifest any pulmonary symptoms. Therefore, GCV was changed to foscarnet (FCV), and the CMV positive cells were eliminated by several days of FCV administration. On day 43, he suddenly complained dyspnea, and chest x-ray and CT revealed the left collapsed lung and multiple pleural bullae in apex of left lung (Figure 1AC), and no sign of pneumomediastinum nor subcutaneous emphysema. Then, we diagnosed as left pneumothorax, and chest drainage tube was inserted. One month later his left lung did not collapse without drainage (Figure 1D, E). Chest CT could not detect any pleural bullae before BMT. During this episode, we could not detect the sign of Pneumocystis pneumonia and aspergillosis infection.

\section{Discussion}

Pneumothorax after HSCT was rare, and reported cases with pneumothorax after HSCT were usually accompanied with pulmonary cGVHD, such as brochiolitis obliterans and
Correspondence: Yasuhiro Ebihara, Division of Stem Cell Processing, Center for Stem Cell Biology and Regenerative Medicine, The Institute of Medical Science, The University of Tokyo, 4-61, Shirokanedai, Minato-ku, Tokyo 108-8639, Japan.

Tel. +81.354.495397 - Fax: +81.354.495428.

E-mail: ebihara@ims.u-tokyo.ac.jp

Key words: pneumothorax, air-leak syndrome, allogeneic hematopoietic stem cell transplantation.

Conflict of interests: the authors declare no potential conflict of interests.

Received for publication: 30 March 2013.

Revision received: 2 May 2013.

Accepted for publication: 21 June 2013.

This work is licensed under a Creative Commons Attribution NonCommercial 3.0 License (CC BYNC 3.0).

(C) Copyright Y. Ebihara et al., 2013

Licensee PAGEPress, Italy

Hematology Reports 2013; 5:e10

doi:10.4081/hr.2013.e10

bronchiolitis obliterans organizing pneumonia. ${ }^{5,6,8}$ The incidence of air leak syndrome (ALS) including pneumothorax, pneumomediastinum and subcutaneous emphysema following HSCT was between 0.83-2.3\%.4-7 ALS occurred within 100 days after HSCT was extremely rare complication. Moon et al. reported 18 cases of ALS including pneumothorax occurred after allogeneic HSCT. ${ }^{6}$ Among them, the onsets of 15 cases were later than 100 days after HSCT (on days 183 to 1825), and only 3 cases occurred earlier than on day 100 (on days 55 to 60 ). In such cases of pneumothorax occurring in an early phase after HSCT, the damages caused by drugs and irradiation therapies in the pulmonary tissues might induce the tissue vulnerability, which could contribute to an easy rupture of alveolar and pleural tissues. Additionally, in the present case, the early onset of aGVHD and the entity of CMV might worsen the tissue damages for the onset of pneumothorax. Interestingly, the recipients with ALS were reported to have poor prognosis. ${ }^{4,5}$ Indeed, our patient died of progression of the primary disease three months after HSCT. Therefore, some of ALS after HSCT might be related to pulmonary infiltration of malignant cells.

\section{Conclusions}

Our experience indicated that we should be aware of the possibility that pneumothorax 


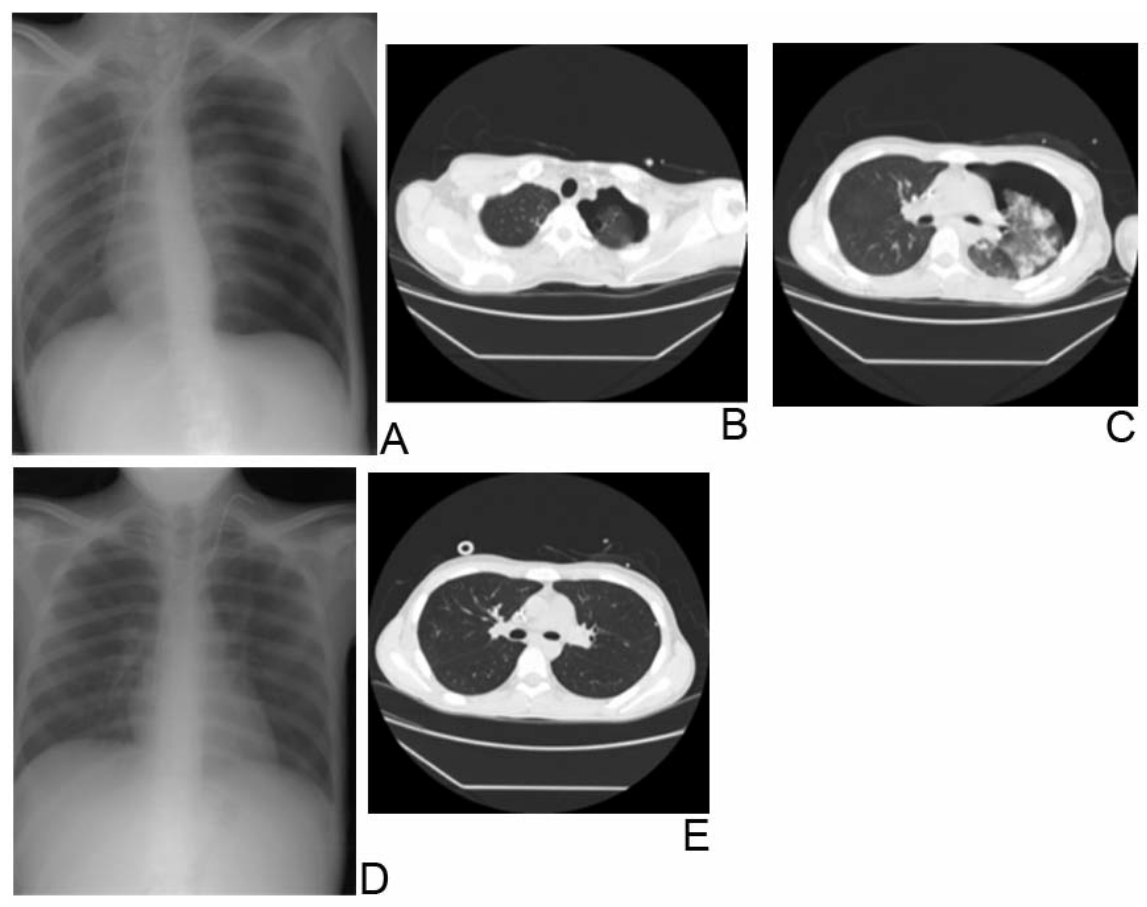

Figure 1. Chest $x$-ray and computed tomography (CT) scan findings. At the onset of symptoms, (A) chest $\mathrm{x}$-ray showed left collapsed lung and pneumothorax, and $(\mathrm{B}, \mathrm{C})$ chest CT scan images showed left collapsed lung and pneumothorax and pleural bullae in appex. One month later, pneumothorax improved without drainage in chest $x$-ray (D) and CT scan (E).

could occur in the early period after allogeneic HSCT although pneumothorax usually develops with the exacerbation of cGVHD in the late period after allogeneic HSCT, and carefully observe the clinical course of the patients even after pneumothorax has been improved.

1. Chadwick C, Marven SM, Vora AJ. Autologous blood pleurodesis for pneumothorax complicating graft-versus-host disease-related bronchiolitis obliterans.
3. Krowka MJ, Rosenow EC 3rd, Hoagland HC. Pulmonary complications of bone marrow transplantation. Chest 1985;87:237-46.

4. Toubai T, Tanaka J, Kobayashi N, et al. Mediastinal emphysema and bilateral pneumothoraces with chronic GVHD in patients after allogeneic stem cell transplantation. Bone Marrow Transplant 2004;33:1159-63.

5. Sakai R, Kanamori H, Nakaseko C, et al. Air-leak syndrome following allo-SCT in adult patients: report from the Kanto Study Group for Cell Therapy in Japan. Bone Marrow Transplant 2011;46:379-84.

6. Moon MH, Sa YJ, Cho KD, et al. Thoracic air-leak syndromes in hematopoietic stem cell transplant recipients with graft-versus-host disease: a possible sign for poor response to treatment and poor prognosis. J Korean Med Sci 2010;25:658-62.

7. Vogel M, Brodoefel $\mathrm{H}$, Bethge $\mathrm{W}$, et al. Spontaneous thoracic air-leakage syndrome in patients following allogeneic hematopoietic stem cell transplantation: causes, CT-follow up and patient outcome. Eur J Radiol 2006;60:392-7.

8. Franquet T, Rodriguez S, Hernandez JM, et al. Air-leak syndromes in hematopoietic stem cell transplant recipients with chronic GVHD: high-resolution CT findings. J Thorac Imaging 2007;22:335-40.

9. Shin HJ, Park CY, Park YH, et al. Spontaneous pneumothorax developed in patients with bronchiolitis obliterans after unrelated hematopoietic stem cell transplantation: case report and review of the literature. Int J Hematol 2004;79:298-302. Bone Marrow Transplant 2004;33:451-3.

2. Quabeck K. The lung as a critical organ in marrow transplantation. Bone Marrow Transplant 1994;14 Suppl 4:S19-28.
10. Kumar S, Tefferi A. Spontaneous pneumomediastinum and subcutaneous emphysema complicating bronchiolitis obliterans after allogeneic bone marrow transplantation-case report and review of literature. Ann Hematol 2001;80:430-5. 